\title{
QUASISYMMETRICALLY MINIMAL MORAN SETS AND HAUSDORFF DIMENSION
}

\author{
Yuxia Dai, Zhixiong Wen, Lifeng Xi and Ying Xiong \\ Huazhong University of Science and Technology, Department of Mathematics \\ Wuhan 430074, P. R. China; daiyuxia8173@163.com \\ Huazhong University of Science and Technology, Department of Mathematics \\ Wuhan 430074, P. R. China; zhi-xiong.wen@mail.hust.edu.cn \\ Zhejiang Wanli University, Institute of Mathematics \\ Ningbo, Zhejiang 315100, P. R. China; xilf@zwu.edu.cn \\ South China University of Technology, Department of Mathematics \\ Guangzhou 510641, P. R. China; xiongyng@gmail.com
}

\begin{abstract}
In this paper, we prove that a large class of Moran sets on the line with Hausdorff dimension 1 are 1-dimensional quasisymmetrically minimal. We also obtain a general theorem on the Hausdorff dimension of Moran set on the line.
\end{abstract}

\section{Introduction}

Let $\left(X, d_{X}\right),\left(Y, d_{Y}\right)$ be metric spaces. A topological homeomorphism $f: X \rightarrow Y$ is called quasisymmetric if there is a homeomorphism $\eta:[0, \infty) \rightarrow[0, \infty)$ such that

$$
\frac{d_{Y}(f(x), f(a))}{d_{Y}(f(x), f(b))} \leq \eta\left(\frac{d_{X}(x, a)}{d_{X}(x, b)}\right)
$$

for all triples $a, b, x$ of distinct points in $X$. In particular, we also say that $f$ is an $n$-dimensional quasisymmetric mapping when $X=Y=\mathbf{R}^{n}$. Quasisymmetry is an important notion in the theory of analysis on metric spaces ([9]) and complex analysis ([2]). It is the generalization of quasiconformality in Euclidean spaces to general metric spaces (see [19]).

Unlike bi-Lipschitz mappings, quasisymmetric mappings do not preserve Hausdorff dimension. So there is a natural problem that how the quasisymmetric mappings change the Hausdorff dimension. Many efforts have been devoted to this problem, especially in Euclidean spaces. For example, if $\operatorname{dim}_{\mathrm{H}} E=0$, then $\operatorname{dim}_{\mathrm{H}} f(E)=0$ for any quasisymmetric mapping $f$, since $f$ is Hölder continuous (see [1]); if $0<\operatorname{dim}_{\mathrm{H}} E<n$, Bishop [3] showed that for any $\varepsilon>0$ there is an $n$-dimensional quasisymmetric mapping $f$ such that $\operatorname{dim}_{\mathrm{H}} f(E)>n-\varepsilon$; Tyson and $\mathrm{Wu}[21]$ obtained that the Sierpinski

doi:10.5186/aasfm.2011.3608

2010 Mathematics Subject Classification: Primary 30C65; Secondary 28A80. measure.

Key words: Quasisymmetrically minimal set, Moran set, Hausdorff dimension, Gibbs-like

This work is supported by National Natural Science Foundation of China (Grant Nos 11071224, 11071082, 11071090, 10671180, 10571140, 10631040, 11071164) the Fundamental Research Funds for the Central Universities, SCUT, the Science Foundation for the Youth of South China University of Technology (E5090470) and Morningside Center of Mathematics.

Ying Xiong is the corresponding author. 
gasket can be mapped by a 2-dimensional quasisymmetric mapping onto a set with Hausdorff dimension arbitrarily close to one.

We call a set $E \subset \mathbf{R}^{n}$ quasisymmetrically minimal if

$$
\operatorname{dim}_{\mathrm{H}} f(E) \geq \operatorname{dim}_{\mathrm{H}} E
$$

for any $n$-dimensional quasisymmetric map $f$. Let $E \subset \mathbf{R}^{n}$ and $f$ be an $n$-dimensional quasisymmetric mapping. The following are some known facts.

(1) Tyson [20] proved that for all $\alpha \in[1, n]$, there exists a set $E \subset \mathbf{R}^{n}$ which is quasisymmetrically minimal with $\operatorname{dim}_{\mathrm{H}} E=\alpha$.

(2) Kovalev [14] pointed out that there is no quasisymmetrically minimal set $E$ with $0<\operatorname{dim}_{\mathrm{H}} E<1$.

(3) If $n \geq 2$, Gehring et al. [6, 7] showed that $n$-dimensional quasisymmetric mappings preserve sets of Hausdorff dimension $n$.

(4) However, when $n=1$, Tukia [18] obtained that there exists $E \subset \mathbf{R}$ of Hausdorff dimension 1 with $\operatorname{dim}_{\mathrm{H}} f(E)<1$ for some 1-dimensional quasisymmetric mapping $f$.

From the above results, we will focus attention on the question that which sets in $\mathbf{R}$ of Hausdorff dimension 1 are minimal. There are some related result:

(1) The first known examples of minimal subsets of $\mathbf{R}$ are quasisymmetrically thick sets. Recall from [17], a set $E \in \mathbf{R}$ is called a quasisymmetrically thick set if $f(E)$ has positive Lebesgue measure for all quasisymmetric mapping $f$.

(2) Hakobyan [8] proved that middle interval Cantor sets of Hausdorff dimension 1 are all minimal. It was shown that these sets need not be quasisymmetrically thick sets.

(3) Recently, $\mathrm{Hu}$ and Wen [10] extended the results in [8]. They proved that uniform Cantor sets of Hausdorff dimension 1 are minimal.

It is worth noting that all minimal sets appearing in $[8,10]$ are some special kinds of Moran sets - homogeneous Cantor sets (see Definition 1 and 2).

In this paper, we will show that the results of $[8,10]$ are no accidents. In fact, a large class of Moran sets on the line with Hausdorff dimension 1 are minimal (Theorem 1). The main tool in the proof of Theorem 1 is some Gibbs-like measures. Moveover, the measures are also useful to determine the Hausdorff dimension of Moran sets. With this measure in hand, we can generalize some classic results in [13] on the Hausdorff dimension of Moran sets (Theorem 2).

This paper is organized as follows. In the rest of Section 1, we state Theorems 1 and 2 in Section 1.1, before the introduction to the Moran sets (Section 1.2). In Section 2, we introduce the so called Gibbs-like measures. The proof of Theorem 1 is given in Section 3, which based on some ideas of Hakobyan [8]. In Section 4.1, we prove Theorem 2. Some remarks on the Hausdorff dimension of Moran sets appear in Section 4.2.

1.1. Main results. With the technical notations and definition of Moran set in Section 1.2, we state our main results. The first one concerns the quasisymmetrically minimal sets on the line. 
Theorem 1. Let $E \in \mathscr{M}\left(J,\left\{n_{k}\right\},\left\{c_{k, j}\right\}\right)$. If $s_{*}=1, \sup _{k} n_{k}<\infty$ and there exists a constant $\alpha \in(0,1)$ such that

$$
\liminf _{n \rightarrow \infty} \frac{\operatorname{card}\left\{1 \leq k \leq n: D_{k} \leq \alpha\right\}}{n}>0,
$$

then $\operatorname{dim}_{\mathrm{H}} E=1$ and $E$ is minimal for 1-dimensional quasisymmetric mapping.

Remark 1. Theorem 1 includes the results in [8, 10]. In fact, [8] requires $n_{k} \equiv 2$, $c_{k, 1}=c_{k, 2}=D_{k}<1 / 2$ for all $k,[10]$ requires $\sup _{k} n_{k}<\infty, c_{k, 1}=\cdots=c_{k, n_{k}}=D_{k} \leq$ $1 / 2$ for all $k$, and all the minimal sets in $[8,10]$ are homogeneous Cantor sets (see Section 1.2) with $s_{*}=1$. In above cases, $s_{*}=1, \sup _{k} n_{k}<\infty$ and (1.1) holds for $\alpha=1 / 2$. Therefore the conditions in Theorem 1 is much weaker than those in $[8,10]$.

As far as we know, there is no theorem to ensure $\operatorname{dim}_{\mathrm{H}} E=1$ under the conditions of Theorem 1. This enlightens a more general theorem on the Hausdorff dimension of Moran sets in the Moran class $\mathscr{M}\left(J,\left\{n_{k}\right\},\left\{c_{k, j}\right\}\right)$.

Theorem 2. Let $E \in \mathscr{M}\left(J,\left\{n_{k}\right\},\left\{c_{k, j}\right\}\right)$. If

$$
\lim _{k \rightarrow \infty} \frac{\log \left(k n_{k}\right)}{-\sum_{i=1}^{k} \log D_{i}}=0
$$

then $\operatorname{dim}_{\mathrm{H}} E=s_{*}$. Moreover, when $s_{*}=1$, the condition that

$$
\sum_{k=1}^{\infty} n_{k}\left(\prod_{i=1}^{k} D_{i}\right)^{\delta}<+\infty \quad \text { for some } \delta \in(0,1)
$$

ensures $\operatorname{dim}_{\mathrm{H}} E=1$.

Remark 2. The condition (1.2) is equivalent to

$$
\sum_{k=1}^{\infty} n_{k}\left(\prod_{i=1}^{k} D_{i}\right)^{\delta}<+\infty \quad \text { for all } \delta>0 .
$$

So the condition (1.3) is weaker than the condition (1.2).

Remark 3. By Theorem 2, it is plain to see that $\operatorname{dim}_{\mathrm{H}} E=1$ under the conditions of Theorem 1. In fact, $\sup _{k} n_{k}<\infty$ implies $\log \left(k n_{k}\right) \sim \log k$ as $k \rightarrow \infty$, and conation (1.1) implies $\sum_{i=1}^{k} \log D_{i}=O(k)$ as $k \rightarrow \infty$. And so the condition of Theorem 2 follows.

Remark 4. In the proof of Theorem 2, we can loosen the restriction $n_{k} \geq 2$ and $c_{k, j} \in(0,1)$ in the definition of Moran sets, here we permit the case that $n_{k}=1$ or $c_{k, j}=1$.

1.2. Definition of Moran sets. Let $\left\{n_{k}\right\}_{k \geq 1}$ be a sequence of positive integers and $\left\{c_{k, j}\right\}_{k \geq 1,1 \leq j \leq n_{k}}$ a sequence of positive numbers satisfying $n_{k} \geq 2$ and $c_{k, j} \in(0,1)$ for all $k \geq 1,1 \leq j \leq n_{k}$. Write

$$
D_{k}=\max _{1 \leq j \leq n_{k}} c_{k, j}, \quad d_{k}=\min _{1 \leq j \leq n_{k}} c_{k, j}, \quad c_{*}=\inf _{k, j} c_{k, j}
$$

and

$$
s_{*}=\liminf _{k \rightarrow \infty} s_{k} \quad \text { and } \quad s^{*}=\limsup _{k \rightarrow \infty} s_{k},
$$


where $s_{k}$ is defined by the equation

$$
\prod_{i=1}^{k} \sum_{j=1}^{n_{i}} c_{i, j}^{s_{k}}=1 .
$$

Let $\Omega_{0}=\{\emptyset\}$, where $\emptyset$ is the empty word. For any positive integer $k$, let

$$
\Omega_{k}=\left\{\left(\sigma_{1}, \ldots, \sigma_{k}\right): \sigma_{j} \in\left[1, n_{j}\right] \cap \mathbf{N} \text { for } 1 \leq j \leq k\right\} .
$$

Define $\Omega=\bigcup_{k \geq 0} \Omega_{k}$. For any integers $l, k$ with $l>k \geq 1$, let

$$
\Omega_{k, l}=\left\{\left(\tau_{k+1}, \ldots, \tau_{l}\right): \tau_{j} \in\left[1, n_{j}\right] \cap \mathbf{N} \text { for } k+1 \leq j \leq l\right\} .
$$

Define $\sigma * \tau=\left(\sigma_{1}, \ldots, \sigma_{k}, \tau_{k+1}, \ldots, \tau_{l}\right) \in \Omega_{l}$ for any $\sigma \in \Omega_{k}$ and $\tau \in \Omega_{k, l}$. The length of $\sigma \in \Omega_{k}$ will be denoted by $|\sigma|(=k)$ and the diameter of set $A \subset \mathbf{R}^{n}$ will be denoted by $|A|$. For convenience, we also use $\sigma_{1} \ldots \sigma_{i}$ to denote $\left(\sigma_{1}, \ldots, \sigma_{i}\right)$.

Definition 1. (Moran set) Suppose that $J \subset \mathbf{R}$ is a closed interval. For a collection $\mathscr{F}=\left\{J_{\sigma}: \sigma \in \Omega\right\}$ of closed subintervals of $J$ with $J_{\emptyset}=J$, we say $\mathscr{F}$ has Moran structure, if for all $k \geq 1$, there are constants $c_{k, 1}, \cdots, c_{k, n_{k}}$ such that for any $\sigma \in \Omega_{k-1}, J_{\sigma * 1}, J_{\sigma * 2}, \cdots, J_{\sigma * n_{k}}$ are subintervals of $J_{\sigma}$ with their interiors pairwise disjoint, and for any $1 \leq j \leq n_{k}$,

$$
\left|J_{\sigma * j}\right| /\left|J_{\sigma}\right|=c_{k, j} .
$$

A Moran set determined by $\mathscr{F}$ is defined by

$$
E(\mathscr{F}):=\bigcap_{k \geq 1} \bigcup_{\sigma \in \Omega_{k}} J_{\sigma} .
$$

Here any $J_{\sigma}$ in $\mathscr{F}$ is called a basic element of $E$. Denote by $\mathscr{M}\left(J,\left\{n_{k}\right\},\left\{c_{k, j}\right\}\right)$ the class of all Moran sets associated with $J,\left\{n_{k}\right\}$ and $\left\{c_{k, j}\right\}$.

Definition 2. (Homogenous Cantor set) We call $E(\mathscr{F})$ a homogeneous Cantor set, if furthermore the Moran structure $\mathscr{F}$ satisfies the following conditions:

(i) for any $k, c_{k, j}$ take the same value $c_{k}$ independent of $j$;

(ii) for any $k \geq 1$ and $\sigma \in \Omega_{k-1}$, the gaps between $J_{\sigma * j}$ and $J_{\sigma *(j+1)}\left(1 \leq j<n_{k}\right)$ are equal;

(iii) for any $k \geq 1$ and $\sigma \in \Omega_{k-1}$, the left endpoint of $J_{\sigma * 1}$ is the same as that of $J_{\sigma}$, and the right endpoint of $J_{\sigma * n_{k}}$ is the same as that of $J_{\sigma}$.

Some special cases of Moran sets were first studied by Moran [15]. The later works $[5,11,12,13,16,22]$ developed the theory on the geometrical structure and dimensions of Moran sets systematically. Roughly speaking, the Moran sets generalize the classic self-similar sets from the following points (see Definition 1):

- the placements of the basic sets at each step of the construction can be arbitrary;

- the contraction ratios may be different at each step;

- the lower limit of the contraction ratios permits zero.

Sometimes, these generalizations make it possible to find a Moran subset $B$ in a given fractal set $A$ with $\operatorname{dim} B=\operatorname{dim} A$. As a result, the theory on the dimensions of Moran sets has become a powerful tool in dimension computation.

When $c_{*}>0$, Hua et al. [11, 12] showed that

$$
\operatorname{dim}_{\mathrm{H}} E=s_{*} \text { and } \operatorname{dim}_{\mathrm{P}} E=\overline{\operatorname{dim}}_{\mathrm{B}} E=s^{*}
$$


for all $E \in \mathscr{M}\left(J,\left\{n_{k}\right\},\left\{c_{k, j}\right\}\right)$ (for $s_{*}$ and $s^{*}$, recall (1.6)). When $c_{*}=0$, Hua et al. [13] also obtained two sufficient conditions under which the dimension formula (1.8) holds (see Theorems A and B in Section 4.2). However, except for the two sufficient conditions, it is little known about the dimensions of Moran sets in the case of $c_{*}=0$. We even don't know what conditions ensure that all Moran sets in $\mathscr{M}\left(J,\left\{n_{k}\right\},\left\{c_{k, j}\right\}\right)$ assume the same Hausdorff (or packing) dimension. It seems very difficult to give a complete answer to this problem. In Theorem 2, we give a more general sufficient condition compared with the two conditions in [13] (see Section 4.2 for details). We hope this result leads to some deep discoveries on the dimensions of Moran sets.

\section{The Gibbs-like measures}

Let $E \in \mathscr{M}\left(J,\left\{n_{k}\right\},\left\{c_{k, j}\right\}\right)$. For every $d \in(0,1)$, by the extension theorem of measures, there exists a unique probability measure $\mu$ supported on $E$ such that $\mu\left(J_{\emptyset}\right)=\mu(E)=1$ and

$$
\mu\left(J_{\sigma * j^{\prime}}\right)=\mu\left(J_{\sigma}\right) \cdot \frac{\left|J_{\sigma * j^{\prime}}\right|^{d}}{\sum_{j=1}^{n_{k}}\left|J_{\sigma * j}\right|^{d}}
$$

for all $k \geq 1, \sigma \in \Omega_{k-1}$ and $1 \leq j^{\prime} \leq n_{k}$. Similarly, for very $d \in(0,1)$ and every 1-dimensional quasisymmetric mapping $f$, there also exists a unique probability measure $\nu$ supported on $f(E)$ such that $\nu\left(f\left(J_{\emptyset}\right)\right)=\nu(f(E))=1$ and

$$
\nu\left(f\left(J_{\sigma * j^{\prime}}\right)\right)=\nu\left(f\left(J_{\sigma}\right)\right) \cdot \frac{\left|f\left(J_{\sigma * j^{\prime}}\right)\right|^{d}}{\sum_{j=1}^{n_{k}}\left|f\left(J_{\sigma * j}\right)\right|^{d}}
$$

for all $k \geq 1, \sigma \in \Omega_{k-1}$ and $1 \leq j^{\prime} \leq n_{k}$.

The measures $\mu$ and $\nu$ are so called Gibbs-like measures. We have some lemmas on the properties of measures $\mu$ and $\nu$.

Lemma 1. Let $k \geq 1$. Suppose that $\sigma \in \Omega_{k}$ and $d \leq s_{k}$, then

$$
\frac{\left|J_{\sigma}\right|^{d}}{\mu\left(J_{\sigma}\right)} \geq \prod_{i=1}^{k} D_{i}^{d-s_{k}}
$$

where $\mu$ is the Gibbs-like measure defined by (2.1).

Proof. By (2.1), we have

$$
\begin{aligned}
\mu\left(J_{\sigma}\right) & =\left|J_{\sigma}\right|^{d} \frac{\left|J_{\sigma_{1} \ldots \sigma_{k-1}}\right|^{d}}{\left|J_{\sigma_{1} \ldots \sigma_{k-1} * 1}\right|^{d}+\cdots+\left|J_{\sigma_{1} \ldots \sigma_{k-1} * n_{k}}\right|^{d}} \cdots \frac{1}{\left|J_{1}\right|^{d}+\cdots+\left|J_{n_{1}}\right|^{d}} \\
& =\left|J_{\sigma}\right|^{d} \frac{1}{c_{k, 1}^{d}+\cdots+c_{k, n_{k}}^{d}} \cdots \frac{1}{c_{1,1}^{d}+\cdots+c_{1, n_{1}}^{d}+\cdots} .
\end{aligned}
$$

By the definition of $s_{k}$ (see (1.7)), we have

$$
\frac{\left|J_{\sigma}\right|^{d}}{\mu\left(J_{\sigma}\right)}=\prod_{i=1}^{k} \sum_{j=1}^{n_{i}} c_{i, j}^{d}=\prod_{i=1}^{k} \frac{\sum_{j=1}^{n_{i}} c_{i, j}^{d}}{\sum_{j=1}^{n_{i}} c_{i, j}^{s_{k}}} \geq \prod_{i=1}^{k} D_{i}^{d-s_{k}}
$$

since $D_{i}=\max _{1 \leq j \leq n_{i}} c_{i, j}$ and $d \leq s_{k}$. 
Let $d \in(0,1)$ and $f$ be a 1-dimensional quasisymmetric mapping. For $k>1$ and $\sigma \in \Omega_{k-1}$, write

$$
\phi_{\sigma}=\frac{\sum_{j=1}^{n_{k}}\left|f\left(J_{\sigma * j}\right)\right|^{d}}{\left(\sum_{j=1}^{n_{k}}\left|f\left(J_{\sigma * j}\right)\right|\right)^{d}} \quad \text { and } \quad \varphi_{\sigma}=\frac{\sum_{j=1}^{n_{k}}\left|f\left(J_{\sigma * j}\right)\right|}{\left|f\left(J_{\sigma}\right)\right|} .
$$

For $\sigma=\emptyset$, write

$$
\phi_{\emptyset}=\frac{\sum_{j=1}^{n_{1}}\left|f\left(J_{j}\right)\right|^{d}}{\left(\sum_{j=1}^{n_{1}}\left|f\left(J_{j}\right)\right|\right)^{d}} \quad \text { and } \quad \varphi_{\emptyset}=\sum_{j=1}^{n_{1}}\left|f\left(J_{j}\right)\right| .
$$

By a similar argument as in the proof of Lemma 1, we have

Lemma 2. Let $d \in(0,1)$ and $f$ be a 1-dimensional quasisymmetric mapping, then for all $k \geq 1$ and $\sigma \in \Omega_{k}$,

$$
\frac{\left|f\left(J_{\sigma}\right)\right|^{d}}{\nu\left(f\left(J_{\sigma}\right)\right)}=\phi_{\emptyset} \varphi_{\emptyset}^{d} \cdot \prod_{i=1}^{k-1} \phi_{\sigma_{1} \ldots \sigma_{i}} \varphi_{\sigma_{1} \ldots \sigma_{i}}^{d}
$$

where $\nu$ is the Gibbs-like measure defined by (2.2).

We need the following lemma to estimate $\phi_{\sigma}$ and $\varphi_{\sigma}$, which is an invariant formulation of Lemma 1 in $\mathrm{Wu}$ [23].

Lemma 3. Let $f$ be a 1-dimensional quasisymmetric mapping. Then

$$
\gamma \frac{|J|^{q}}{|I|^{q}} \leq \frac{|f(J)|}{|f(I)|} \leq 4 \frac{|J|^{p}}{|I|^{p}}
$$

for all intervals $I, J$ with $J \subset I$, where $\gamma, p, q$ are three constants dependent on $f$ with $\gamma>0,0<p \leq 1 \leq q$.

Lemmas 4 and 5 are devoted to the lower bound of $\phi_{\sigma}$ and $\varphi_{\sigma}$.

Lemma 4. Let $k \geq 1, \sigma \in \Omega_{k-1}$ and $\phi_{\sigma}$ as in (2.3) and (2.4), then $\phi_{\sigma}>1$. Moreover, if $\sum_{j=1}^{n_{k}} c_{k, j} \geq \beta>\alpha \geq D_{k}$ for some constants $\alpha, \beta$ with $1>\beta>\alpha>0$, then $\phi_{\sigma}>1+\epsilon$, where $\epsilon>0$ is a constant dependent on $\alpha, \beta, n_{k}$ and $f$.

Proof. Since $d \in(0,1)$, it is obvious that $\phi_{\sigma}>1$. Now suppose that $\sum_{j=1}^{n_{k}} c_{k, j} \geq$ $\beta>\alpha \geq D_{k}$. Without loss of generality, we assume that

$$
M_{\sigma}=\max _{1 \leq j \leq n_{k}}\left|f\left(J_{\sigma * j}\right)\right|=\left|f\left(J_{\sigma * 1}\right)\right| .
$$

Write

$$
x=\frac{\sum_{j=2}^{n_{k}}\left|f\left(J_{\sigma * j}\right)\right|}{M_{\sigma}} .
$$

By Lemma 3,

$$
\frac{\sum_{j=2}^{n_{k}}\left|f\left(J_{\sigma * j}\right)\right|}{\left|f\left(J_{\sigma}\right)\right|} \geq \gamma \sum_{j=2}^{n_{k}} c_{k, j}^{q} \geq \gamma\left(n_{k}-1\right)^{1-q}\left(\sum_{j=2}^{n_{k}} c_{k, j}\right)^{q} \geq \frac{\gamma(\beta-\alpha)^{q}}{n_{k}^{q}}
$$

and $M_{\sigma} \cdot\left|f\left(J_{\sigma}\right)\right|^{-1} \leq 4 D_{k}^{p} \leq 4 \alpha^{p}$. Therefore,

$$
x \geq \frac{\gamma(\beta-\alpha)^{q}}{4 \alpha^{p} n_{k}^{q}}>0 .
$$


We return to estimate $\phi_{\sigma}$. Since $\left|f\left(J_{\sigma * j}\right)\right| \cdot M_{\sigma}^{-1} \leq 1$ for $1 \leq j \leq n_{k}$, we have

$$
\phi_{\sigma}=\frac{\sum_{j=1}^{n_{k}}\left|f\left(J_{\sigma * j}\right)\right|^{d} \cdot M_{\sigma}^{-d}}{\left(\sum_{j=1}^{n_{k}}\left|f\left(J_{\sigma * j}\right)\right| \cdot M_{\sigma}^{-1}\right)^{d}} \geq \frac{\sum_{j=1}^{n_{k}}\left|f\left(J_{\sigma * j}\right)\right| \cdot M_{\sigma}^{-1}}{\left(\sum_{j=1}^{n_{k}}\left|f\left(J_{\sigma * j}\right)\right| \cdot M_{\sigma}^{-1}\right)^{d}}=(1+x)^{1-d} .
$$

Together with (2.5), we complete the proof.

Lemma 5. Let $k>1, \sigma \in \Omega_{k-1}$ and $\varphi_{\sigma}$ as in (2.3). Let $\gamma, p, q$ be as in Lemma 3, then $\varphi_{\sigma} \geq \gamma n_{k}^{1-q}\left(\sum_{j=1}^{n_{k}} c_{k, j}\right)^{q}$. Moreover, when $\sum_{j=1}^{n_{k}} c_{k, j}$ is sufficiently close to 1 , the following lower bound is useful:

$$
\varphi_{\sigma} \geq 1-8 n_{k}\left(1-\sum_{j=1}^{n_{k}} c_{k, j}\right)^{p}
$$

Proof. By Lemma 3 and (2.3), it follows that

$$
\varphi_{\sigma} \geq \gamma \sum_{j=1}^{n_{k}} c_{k, j}^{q} \geq \gamma n_{k}^{1-q}\left(\sum_{j=1}^{n_{k}} c_{k, j}\right)^{q} .
$$

We now turn to the other lower bound. Let $\Delta_{0}, \ldots, \Delta_{n_{k}}$ (some $\Delta_{j}$ may be empty) be the connected components of $J_{\sigma} \backslash \bigcup_{j=1}^{n_{k}} J_{\sigma * j}$ and $\delta_{j}=\left|\Delta_{j}\right| /\left|J_{\sigma}\right|$. Then $\sum_{j=1}^{n_{k}} c_{k, j}+$ $\sum_{j=0}^{n_{k}} \delta_{j}=1$. Together with Lemma 3 , we have

$$
\begin{aligned}
\varphi_{\sigma} & =\frac{\sum_{j=1}^{n_{k}}\left|f\left(J_{\sigma * j}\right)\right|}{\left|f\left(J_{\sigma}\right)\right|}=\frac{\left|f\left(J_{\sigma}\right)\right|-\sum_{j=0}^{n_{k}}\left|f\left(\Delta_{j}\right)\right|}{\left|f\left(J_{\sigma}\right)\right|} \geq 1-4 \sum_{j=0}^{n_{k}} \delta_{j}^{p} \\
& \geq 1-4\left(n_{k}+1\right)\left(\sum_{j=0}^{n_{k}} \delta_{j}\right)^{p} \geq 1-8 n_{k}\left(1-\sum_{j=1}^{n_{k}} c_{k, j}\right)^{p} .
\end{aligned}
$$

\section{1-dimensional quasisymmetrically minimal set}

This section is devoted to the proof of Theorem 1. We begin with some lemmas.

Lemma 6. Suppose that $\lim _{k \rightarrow \infty} s_{k}=1$ and $\sup _{k} n_{k}<\infty$. Then

(a) $\lim _{k \rightarrow \infty} k^{-1} \sum_{i=1}^{k} \log \sum_{j=1}^{n_{i}} c_{i, j}=0$;

(b) $\lim _{k \rightarrow \infty} k^{-1} \operatorname{card}\left\{1 \leq i \leq k: \sum_{j=1}^{n_{i}} c_{i, j}<\beta\right\}=0$ for any $\beta \in(0,1)$;

(c) $\lim _{k \rightarrow \infty} k^{-1} \sum_{i=1}^{k}\left(1-\sum_{j=1}^{n_{i}} c_{i, j}\right)^{p}=0$ for any $p>0$.

Proof. (a) By the Jensen's inequality,

$$
n_{i}^{-1} \sum_{j=1}^{n_{i}} c_{i, j} \geq\left(n_{i}^{-1} \sum_{j=1}^{n_{i}} c_{i, j}^{s_{k}}\right)^{1 / s_{k}} \quad \text { for all } 1 \leq i \leq k,
$$

since $s_{k} \leq 1$. By $(1.7)$, a simple computation shows that

$$
\frac{1}{k} \sum_{i=1}^{k} \log \sum_{j=1}^{n_{i}} c_{i, j} \geq \frac{\sum_{i=1}^{k} \log n_{i}}{k}\left(1-s_{k}^{-1}\right) \geq\left(1-s_{k}^{-1}\right) \cdot \log \sup _{i \geq 1} n_{i} .
$$

Together with $\sum_{j=1}^{n_{i}} c_{i, j} \leq 1$ and $\lim _{k \rightarrow \infty} s_{k}=1$, we have

$$
0 \geq \lim _{k \rightarrow \infty} \frac{1}{k} \sum_{i=1}^{k} \log \sum_{j=1}^{n_{i}} c_{i, j} \geq \lim _{k \rightarrow \infty}\left(1-s_{k}^{-1}\right) \cdot \log \sup _{i \geq 1} n_{i}=0 .
$$


(b) It follows immediately from (a).

(c) For any $\beta \in(0,1)$, we have

$$
\frac{1}{k} \sum_{i=1}^{k}\left(1-\sum_{j=1}^{n_{i}} c_{i, j}\right)^{p} \leq(1-\beta)^{p}+\frac{1}{k} \operatorname{card}\left\{1 \leq i \leq k: \sum_{j=1}^{n_{i}} c_{i, j}<\beta\right\} .
$$

Then (c) follows from (b) and the arbitrariness of $\beta$.

Lemma 7. Let $d \in(0,1)$ and $f$ be a 1-dimensional quasisymmetric mapping. Let $\nu$ be the Gibbs-like measure defined by (2.2). Suppose that the conditions in Theorem 1 hold, then there exists a constant $\zeta>0$ such that for sufficiently large $k$,

$$
\frac{\left|f\left(J_{\sigma}\right)\right|^{d}}{\nu\left(f\left(J_{\sigma}\right)\right)}>(1+\zeta)^{k} \quad \text { for all } \sigma \in \Omega_{k}
$$

Proof. Let $\alpha$ be as in (1.1). Pick a $\beta \in(\alpha, 1)$. Then by (1.1) and Lemma 6 (b),

$$
\liminf _{k \rightarrow \infty} \frac{1}{k} \operatorname{card}\left\{1 \leq i \leq k: \sum_{j=1}^{n_{i}} c_{i, j} \geq \beta>\alpha \geq D_{i}\right\}>\lambda>0 .
$$

Together with Lemma 4 and $\sup _{i \geq 1} n_{i}<\infty$, it follows that there exists $\epsilon>0$ such that for sufficiently large $k$,

$$
\prod_{i=0}^{k-1} \phi_{\sigma_{1} \ldots \sigma_{i}}>(1+\epsilon)^{\lambda k} \quad \text { for all } \sigma \in \Omega_{k} .
$$

By Lemma 2 and (3.1), to complete the proof, it suffices to show that for every $\varepsilon>0$,

$$
\varepsilon>\frac{1}{k} \sum_{i=0}^{k-1} \log \varphi_{\sigma_{1} \ldots \sigma_{i}}>-\varepsilon
$$

for sufficiently large $k$ and all $\sigma \in \Omega_{k}$.

The left inequality is obvious since $\varphi_{\tau} \leq 1$ for all $\tau \neq \emptyset$. For the right one, we apply Lemma 5 . Since $\sup _{i} n_{i}<\infty$, we can pick a $\beta^{\prime} \in(0,1)$ such that

$$
8 \sup _{i \geq 1} n_{i} \cdot\left(1-\beta^{\prime}\right)^{p}<\frac{1}{2},
$$

where $p$ is as in (2.6). For $k \geq 1$, write

$$
\Lambda_{k}=\left\{1 \leq i \leq k: \sum_{j=1}^{n_{i}} c_{i, j} \geq \beta^{\prime}\right\} \quad \text { and } \quad \Lambda_{k}^{*}=\left\{1 \leq i \leq k: \sum_{j=1}^{n_{i}} c_{i, j}<\beta^{\prime}\right\} .
$$

Then by Lemma 5 , for every $\sigma \in \Omega_{k}$, we have

$$
\begin{aligned}
& \frac{1}{k} \sum_{i \in \Lambda_{k}^{*}} \log \varphi_{\sigma_{1} \ldots \sigma_{i-1}} \geq \frac{1}{k} \sum_{i \in \Lambda_{k}^{*}} \log \left(\gamma n_{i}^{1-q}\left(\sum_{j=1}^{n_{i}} c_{i, j}\right)^{q}\right) \\
& \geq \frac{1}{k} \operatorname{card} \Lambda_{k}^{*} \cdot\left(\log \gamma+(1-q) \log \sup _{i \geq 1} n_{i}\right)+\frac{q}{k} \sum_{i=1}^{k} \log \sum_{j=1}^{n_{i}} c_{i, j} \rightarrow 0,
\end{aligned}
$$


as $k \rightarrow+\infty$, according to (b) and (a) of Lemma 6 . Also by Lemma 5 , for every $\sigma \in \Omega_{k}$

$$
\begin{aligned}
& \frac{1}{k} \sum_{i \in \Lambda_{k}} \log \varphi_{\sigma_{1} \ldots \sigma_{i-1}} \geq \frac{1}{k} \sum_{i \in \Lambda_{k}} \log \left(1-8 n_{i}\left(1-\sum_{j=1}^{n_{i}} c_{i, j}\right)^{p}\right) \\
& \geq-\frac{16}{k} \sum_{i \in \Lambda_{k}} n_{i}\left(1-\sum_{j=1}^{n_{i}} c_{i, j}\right)^{p} \geq-16 \sup _{i \geq 1} n_{i} \cdot \frac{1}{k} \sum_{i=1}^{k}\left(1-\sum_{j=1}^{n_{i}} c_{i, j}\right)^{p} \rightarrow 0
\end{aligned}
$$

as $k \rightarrow+\infty$, according to (c) of Lemma 6 . The second inequality of above estimation follows from (3.3) and the fact that $\log (1-t) \geq-2 t$ for all $t \in(0,1 / 2)$. Inequalities (3.4) and (3.5) implies the right inequality of (3.2), and so the proof is completed.

The following lemma concerns the geometrical structure of $f(E)$.

Lemma 8. Let $U$ be an interval. For $k \geq 1$, write

$$
\Theta_{k}^{U}=\left\{\sigma \in \Omega_{k}: f\left(J_{\sigma}\right) \subset U \text { and } f\left(J_{\sigma_{1} \ldots \sigma_{k-1}}\right) \not \subset U\right\},
$$

then card $\Theta_{k}^{U} \leq 2 n_{k}$.

Proof. Write $\Theta=\left\{\tau \in \Omega_{k-1}: f\left(J_{\tau}\right) \cap U \neq \varnothing\right.$ and $\left.f\left(J_{\tau}\right) \not \subset U\right\}$, then

$$
\Theta_{k}^{U} \subset\left\{\tau * j: \tau \in \Theta \text { and } 1 \leq j \leq n_{k}\right\} \text {. }
$$

Therefore, we only need to show that card $\Theta \leq 2$. If otherwise, suppose that $\tau^{1}, \tau^{2}, \tau^{3} \in \Theta$ and the position of $f\left(J_{\tau^{1}}\right), f\left(J_{\tau^{2}}\right)$ and $f\left(J_{\tau^{3}}\right)$ are from left to right. Since $f\left(J_{\tau^{1}}\right) \cap U \neq \varnothing, f\left(J_{\tau^{3}}\right) \cap U \neq \varnothing$ and $U$ is an interval, we must have $f\left(J_{\tau^{2}}\right) \subset U$. Contradiction!

We are now ready for the proof of Theorem 1.

The proof of Theorem 1. It suffices to prove that $\operatorname{dim}_{\mathrm{H}} f(E) \geq d$ for all $d \in(0,1)$ and all 1-dimensional quasisymmetric mapping $f$. To this end, fix $d$ and $f$, let $\nu$ be the Gibbs-like measure defined by (2.2). We will show that there exists a constant $c>0$ such that

$$
\nu(U) \leq c|U|^{d} \text { for all interval } U .
$$

Then the conclusion $\operatorname{dim}_{H} f(E) \geq d$ follows from the mass distribution principle (see [4, Proposition 2.1]).

By Lemma 7 , there exists a constant $c_{0}>0$ such that

$$
\nu\left(f\left(J_{\sigma}\right)\right) \leq c_{0} \cdot \frac{\left|f\left(J_{\sigma}\right)\right|^{d}}{(1+\zeta)^{|\sigma|}} \quad \text { for all } \sigma \in \Omega .
$$

Let $U$ be an interval and $\Theta_{k}^{U}$ as in Lemma 8, then

$$
\begin{aligned}
\nu(U) & =\nu\left(\bigcup_{k \geq 0} \bigcup_{\sigma \in \Theta_{k}^{U}} f\left(J_{\sigma}\right)\right)=\sum_{k \geq 0} \sum_{\sigma \in \Theta_{k}^{U}} \nu\left(f\left(J_{\sigma}\right)\right) \leq c_{0} \cdot \sum_{k \geq 0} \sum_{\sigma \in \Theta_{k}^{U}} \frac{\left|f\left(J_{\sigma}\right)\right|^{d}}{(1+\zeta)^{k}} \\
& \leq c_{0} \cdot \sum_{k \geq 0} \frac{\left(\operatorname{card} \Theta_{k}^{U}\right)^{1-d}\left(\sum_{\sigma \in \Theta_{k}^{U}}\left|f\left(J_{\sigma}\right)\right|\right)^{d}}{(1+\zeta)^{k}}
\end{aligned}
$$




$$
\begin{aligned}
& \leq c_{0}\left(2 \sup _{i \geq 1} n_{i}\right)^{1-d} \sum_{k \geq 0} \frac{\left(\sum_{\sigma \in \Theta_{k}^{U}}\left|f\left(J_{\sigma}\right)\right|\right)^{d}}{(1+\zeta)^{k}} \\
& \leq c_{0}\left(2 \sup _{i \geq 1} n_{i}\right)^{1-d}\left(\sum_{k \geq 0}(1+\zeta)^{-k /(1-d)}\right)^{1-d}\left(\sum_{k \geq 0} \sum_{\sigma \in \Theta_{k}^{U}} f\left(J_{\sigma}\right)\right)^{d} \leq c|U|^{d} .
\end{aligned}
$$

The second and the fourth inequality of the above estimation follow from the Hölder inequality. Thus we complete the proof.

\section{The Hausdorff dimension of Moran set}

In this section, we will prove Theorem 2 and give some remarks on the Hausdorff dimension of Moran set.

4.1. The proof of Theorem 2. We begin with a lemma similar to Lemma 8 .

Lemma 9. Let $U$ be an interval. For $k \geq 1$, write

$$
\Xi_{k}^{U}=\left\{\sigma \in \Omega_{k}: J_{\sigma} \subset U \text { and } J_{\sigma_{1} \ldots \sigma_{k-1}} \not \subset U\right\},
$$

then card $\Xi_{k}^{U} \leq 2 n_{k}$.

Proof of Theorem 2. According to [13, Proposition 2.1], the statement $\operatorname{dim}_{\mathrm{H}} E \leq$ $s_{*}$ is true. This implies that Theorem 2 holds when $s_{*}=0$. So we may assume $s_{*}>0$ and only need to show that $\operatorname{dim}_{\mathrm{H}} E \geq d$ for all $d \in\left(0, s_{*}\right)$. For this, fix $d \in\left(0, s_{*}\right)$, let $\mu$ be the Gibbs-like measure defined by (2.1). We will show that

$$
\mu(U) \leq c|U|^{d} \text { for all interval } U \text { with }|U| \text { small enough, }
$$

where $c>0$ is a constant. Then by the mass distribution principle, we obtain $\operatorname{dim}_{\mathrm{H}} E \geq d$ and the proof is completed.

Since $d<s_{*}$, for every $\delta \in(0,1)$, there is an integer $K_{\delta}$ such that $\delta\left(s_{*}-d\right) \leq s_{k}-d$ for all $k \geq K_{\delta}$. Together with Lemma 1 , it follows that

$$
\mu\left(J_{\sigma}\right) \leq\left|J_{\sigma}\right|^{d} \cdot \prod_{i=1}^{|\sigma|} D_{i}^{\delta\left(s_{*}-d\right)} \quad \text { for all } \sigma \in \bigcup_{k \geq K_{\delta}} \Omega_{k} .
$$

Let $U$ be an interval with $|U|<\min _{\sigma \in \Omega_{K_{\delta}}}\left|J_{\sigma}\right|$ and $\Xi_{k}^{U}$ as in Lemma 9 , then

$$
\begin{aligned}
\mu(U) & =\mu\left(\bigcup_{k>K_{\delta}} \bigcup_{\sigma \in \Xi_{k}^{U}} J_{\sigma}\right)=\sum_{k>K_{\delta}} \sum_{\sigma \in \Xi_{k}^{U}} \mu\left(J_{\sigma}\right) \leq \sum_{k>K_{\delta}} \sum_{\sigma \in \Xi_{k}^{U}}\left(\left|J_{\sigma}\right|^{d} \prod_{i=1}^{k} D_{i}^{\delta\left(s_{*}-d\right)}\right) \\
& \leq \sum_{k>K_{\delta}}\left(\left(\operatorname{card} \Xi_{k}^{U}\right)^{1-d} \cdot\left(\sum_{\sigma \in \Xi_{k}^{U}}\left|J_{\sigma}\right|\right)^{d} \cdot \prod_{i=1}^{k} D_{i}^{\delta\left(s_{*}-d\right)}\right) \\
& \leq 2^{1-d} \sum_{k>K_{\delta}}\left(n_{k}^{1-d} \prod_{i=1}^{k} D_{i}^{\delta\left(s_{*}-d\right)} \cdot\left(\sum_{\sigma \in \Xi_{k}^{U}}\left|J_{\sigma}\right|\right)^{d}\right)
\end{aligned}
$$




$$
\begin{aligned}
& \leq 2^{1-d}\left(\sum_{k>K_{\delta}} n_{k} \prod_{i=1}^{k} D_{i}^{\frac{\delta\left(s_{*}-d\right)}{1-d}}\right)^{1-d}\left(\sum_{k>K_{\delta}} \sum_{\sigma \in \Xi_{k}^{U}}\left|J_{\sigma}\right|\right)^{d} \\
& \leq 2^{1-d}\left(\sum_{k>K_{\delta}} n_{k} \prod_{i=1}^{k} D_{i}^{\frac{\delta\left(s_{*}-d\right)}{1-d}}\right)^{1-d} \cdot|U|^{d} .
\end{aligned}
$$

The Hölder inequality is used in the second and the fourth inequality of above computation.

When $s_{*}=1$, taking $\delta$ as in (1.3), we obtain $\mu(U) \leq c|U|^{d}$ for $U$ with $|U|$ small enough. For general $s_{*}$, since $s_{*}-d$ can be arbitrarily small, we must require the condition (1.4) to ensure $\mu(U) \leq c \mid U^{d}$.

It remains to show that the condition (1.4) is equivalent to (1.2). When (1.2) holds,

$$
\sum_{k=1}^{\infty} n_{k}\left(\prod_{i=1}^{k} D_{i}\right)^{\delta}<c_{\delta} \sum_{k=1}^{\infty} n_{k} \cdot\left(k n_{k}\right)^{-2}<\infty \quad \text { for all } \delta>0
$$

Conversely, suppose that (1.4) is true. Then $n_{k} \prod_{i=1}^{k} D_{i}^{\delta} \rightarrow 0$ for all $\delta>0$, and so

$$
\lim _{k \rightarrow \infty} \frac{\log n_{k}}{-\sum_{i=1}^{k} \log D_{i}}=0
$$

Noting that the sequence $\left\{\prod_{i=1}^{k} D_{i}^{\delta}\right\}_{k}$ is decreasing and summable for all $\delta>0$, so we have $\prod_{i=1}^{k} D_{i}^{\delta}<k^{-1}$ when $k$ large enough. It follows that

$$
\lim _{k \rightarrow \infty} \frac{\log k}{-\sum_{i=1}^{k} \log D_{i}}=0
$$

Therefore, (1.4) implies (1.2).

4.2. Some remarks. When $c_{*}=0$, there are two theorems which concern the dimensions of Moran sets obtained in [13].

Theorem A. Let $\mathscr{M}=\mathscr{M}\left(J,\left\{n_{k}\right\},\left\{c_{k, j}\right\}\right)$ be a Moran class. Suppose that

(i) $\sup _{k} n_{k}<\infty$;

(ii) $0<\inf _{i} D_{i} \leq \sup _{i} D_{i}<1$.

Then for all $E \in \mathscr{M}$, dimension formula (1.8) holds.

Theorem B. Let $\mathscr{M}=\mathscr{M}\left(J,\left\{n_{k}\right\},\left\{c_{k, j}\right\}\right)$ be a Moran class. Suppose that

$$
\lim _{k \rightarrow \infty} \frac{\log d_{k}}{\sum_{i=1}^{k} \log D_{i}}=0
$$

then for all $E \in \mathscr{M}$, dimension formula (1.8) holds.

We will show that the conditions in Theorem A and B both imply the condition (1.2). For the conditions of Theorem A, it is easy to check. For Theorem B, since $n_{k} d_{k} \leq \sum_{j=1}^{n_{k}} c_{k, j} \leq 1$, we have $\lim _{k \rightarrow \infty} \frac{\log n_{k}}{-\sum_{i=1}^{k} \log D_{i}}=0$. So it remains to show that

$$
\lim _{k \rightarrow \infty} \frac{\log k}{-\sum_{i=1}^{k} \log D_{i}}=0
$$


If otherwise, suppose that there exist a constant $c>0$ and a sequence $\left\{k_{j}\right\}_{j \geq 1}$ such that

$$
\frac{\log k_{j}}{-\sum_{i=1}^{k_{j}} \log D_{i}}>c
$$

Then we can find $K>0$ such that

$$
\frac{\log d_{k}}{\sum_{i=1}^{k} \log D_{i}}<\frac{c}{2}<\frac{\log k_{j}}{-2 \sum_{i=1}^{k_{j}} \log D_{i}} \text { for all } k \geq K \text { and all } k_{j} .
$$

Thus for all $K \leq k \leq k_{j}$, we have $d_{k} \geq k_{j}^{-1 / 2}$, and so $D_{k} \leq 1-d_{k} \leq 1-k_{j}^{-1 / 2}$. It follows that for all $k_{j} \geq 2 K$,

$$
\frac{\log k_{j}}{-\sum_{i=1}^{k_{j}} \log D_{i}} \leq \frac{\log k_{j}}{-\sum_{i=K+1}^{k_{j}} \log D_{i}} \leq \frac{\log k_{j}}{-\sum_{i=K+1}^{k_{j}} \log \left(1-k_{j}^{-1 / 2}\right)} \leq \frac{2 \log k_{j}}{k_{j}^{1 / 2}} \rightarrow 0,
$$

as $k_{j} \rightarrow \infty$. This contradicts (4.1).

By above discussion, we see that Theorem 2 generalizes Theorem A and B in some sense.

\section{References}

[1] Ahlfors, L. V.: Lectures on quasiconformal mappings. - Univ. Lecture Ser. 38, American Mathematical Society, Providence, RI, second edition, 2006.

[2] Beurling, A., and L. Ahlfors: The boundary correspondence under quasiconformal mappings. - Acta Math. 96, 1956, 125-142.

[3] Bishop, C. J.: Quasiconformal mappings which increase dimension. - Ann. Acad. Sci. Fenn. Math. 24:2, 1999, 397-407.

[4] Falconer, K.: Techniques in fractal geometry. - John Wiley \& Sons Ltd., Chichester, 1997.

[5] Feng, D. J., Z. Y. Wen, and J. Wu: Some dimensional results for homogeneous Moran sets. - Sci. China Ser. A 40:5, 1997, 475-482.

[6] Gehring, F. W.: The $L^{p}$-integrability of the partial derivatives of a quasiconformal mapping. - Acta Math. 130, 1973, 265-277.

[7] Gehring, F. W., and J. VÄıs̈̈LÄ: Hausdorff dimension and quasiconformal mappings. - J. London Math. Soc. (2) 6, 1973, 504-512.

[8] Hakobyan, H.: Cantor sets that are minimal for quasi-symmetric mappings. - J. Contemp. Math. Anal. 41:2, 2006, 13-21.

[9] Heinonen, J.: Lectures on analysis on metric spaces. - Universitext, Springer-Verlag, New York, 2001.

[10] Hu, M. D., and S. Y. Wen: Quasisymmetrically minimal uniform Cantor sets. - Topology Appl. 155:6, 2008, 515-521.

[11] HuA, S.: The dimensions of generalized self-similar sets. - Acta Math. Appl. Sin. 17:4, 1994, $551-558$.

[12] HuA, S., and W.X. Li: Packing dimension of generalized Moran sets. - Progr. Natur. Sci. (English Ed.) 6:2, 1996, 148-152.

[13] Hua, S., H. RaO, Z. Y. Wen, and J. Wu: On the structures and dimensions of Moran sets. - Sci. China Ser. A 43:8, 2000, 836-852.

[14] Kovalev, L. V.: Conformal dimension does not assume values between zero and one. - Duke Math. J. 134:1, 2006, 1-13. 
[15] Moran, P. A. P.: Additive functions of intervals and Hausdorff measure. - Proc. Cambridge Philos. Soc. 42, 1946, 15-23.

[16] RAO, H., Z. Y. Wen, and J. Wu: Net measure properties of Moran sets and applications. Chinese Sci. Bull. 43:5, 1998, 386-389.

[17] Staples, S. G., and L. A. Ward: Quasisymmetrically thick sets. - Ann. Acad. Sci. Fenn. Math. 23:1, 1998, 151-168.

[18] Tukia, P.: Hausdorff dimension and quasisymmetric mappings. - Math. Scand. 65:1, 1989, $152-160$.

[19] Tyson, J.: Quasiconformality and quasisymmetry in metric measure spaces. - Ann. Acad. Sci. Fenn. Math. 23:2, 1998, 525-548.

[20] Tyson, J. T.: Sets of minimal Hausdorff dimension for quasiconformal maps. - Proc. Amer. Math. Soc. 128:11, 20000, 3361-3367.

[21] Tyson, J. T., and J. M. Wu: Quasiconformal dimensions of self-similar fractals. - Rev. Mat. Iberoamericana 22:1, 2006, 205-258.

[22] Wen, Z. Y.: Moran sets and Moran classes. - Chinese Sci. Bull. 46:22, 2001, 1849-1856.

[23] Wu, J. M.: Null sets for doubling and dyadic doubling measures. - Ann. Acad. Sci. Fenn. Ser. A I Math. 18:1, 1993, 77-91.

Received 22 January 2010 